\title{
UWB Joint TOA and DOA Estimation
}

\author{
Eva Lagunas*, Montse Najar*†, Monica Navarro ${ }^{\dagger}$ \\ *Universitat Politecnica de Catalunya (UPC), Barcelona, Spain \\ ${ }^{\dagger}$ Centre Tecnologic de Comunicacions de Catalunya (CTTC), Castelldefels, Spain \\ Email: elagunas@gps.tsc.upc.edu, montse.najar@upc.edu, monica.navarro@cttc.cat
}

\begin{abstract}
This paper deals with the simultaneous estimation of Time of Arrival (TOA) and Direction of Arrival (DOA) in UWB systems considering multipath propagation. The estimation is performed in the frequency domain by computing a two dimensional power delay-angle spectrum based on the periodogram because of its low computational complexity. High accuracy is obtained in the estimation of both parameters, TOA and DOA, based on the wide bandwidth of the UWB signals.
\end{abstract}

\section{INTRODUCTION}

One interesting problem in wireless communications is the estimation of the angle of incidence and time of arrival of a signal arriving at a base station antenna array, assuming that a specular multipath channel model holds true. This problem has several applications, including, for example, positioning systems.

UWB radio is a fast emerging technology with uniquely attractive features inviting major advances in wireless communications, networking, radar, imaging, and also positioning systems. This radio technology can potentially enable very accurate ranging and location applications, given the extremely short duration of the pulses. Our method exploits this high time resolution nature of the UWB signals to estimate simultaneous TOA and DOA.

Joint estimation of angle and delay has been widely investigated for narrowband systems.

In comparison with 'classical' disjoint techniques [1]- [2], which first estimates the angle of arrive and, sequentially, the time of arrival corresponding to the previous angle, joint estimation has an advantage in cases where multiple rays have approximately equal delays (or angles).

Parametric joint angle/delay estimation has received increased research interest lately. Methods for joint angle/delay estimation (JADE) were proposed in [3]- [4]. In JADE, paths can be resolved when they are separated in at least one of the two domains, either angle or time. JADE in general does not require more antennas than paths present (as is needed for identifiability in DOA models).

In a parametric multipath propagation model, a source is received by an antenna array via a number of rays, each described by an arrival angle, a delay, and a fading parameter. Unlike the fading, the angles and delays are stationary over long time intervals. This idea is exploited in many approaches

\footnotetext{
${ }^{1}$ This work is partially supported by the Ministry of Science and Education TEC2008-06327 (jointly financed by FEDER) and the Network of Excellence NEWCOM++.
}

such as [4] where the algorithm used is based on a multidimensional version of ESPRIT. Previously, algorithms like MLE [5] or multidimensional MUSIC [6] were used but were discarded because they are unattractive for online estimation.

This paper focus on the joint estimation of the time of arrival of the first path and the corresponding angle of arrival of a single source received by an antenna array in a very wide band system.

The majority of the localization proposals in UWB systems are based on time delay estimation with few contributions considering the direction of arrival. In [7] the authors consider the specific problem of DOA estimation in a large bandwidth system for the ideal channel. In [8] DOA estimation is performed using a digital channelization receiver that assumes complete knowledge of the channel.

In a previous work, the authors exploited the fact that the temporal delay at each antenna element depends not only on the propagation delay but on the direction of arrival. In [10] the proposed estimation technique considers a frequency domain approach. Working in the frequency domain facilitates the application of high resolution spectral estimation techniques to obtain a signal power profile where the TOA is estimated for each array antenna. Then, the DOA estimation is obtained from the previous TOA measurements by means a linear estimation. In this paper the authors propose to develop a two dimensional signal power profile from which the joint estimation of both parameters, angle and delay, are obtained. The two dimensional power profile is defined as the signal energy distribution with respect to the propagation delays in one dimension, and the signal energy distribution with respect to the angles of arrival in the second dimension. The spectral estimation is based on the periodogram aiming at a low complexity implementation and assumes no knowledge of the received pulse waveform.

The outline of the paper is as follows: Section II introduces the IR-UWB signal model for nodes equipped with an antenna array. Section III describes the proposed joint TOA and DOA estimation scheme. Finally, numerical results and conclusions are discussed in Section IV and V, respectively.

\section{SYSTEM MODEL}

We consider an IR-UWB system where transmission of an information symbol is typically implemented by the repetition of $N_{f}$ pulses of very short duration. The transmitted signal is 
expressed as,

$$
s(t)=\sum_{k=-\infty}^{\infty} \sum_{j=0}^{N_{f}-1} p\left(t-T_{j}^{k}\right)
$$

where $T_{j}^{k}=\left(k N_{f}+j\right) T_{f}+c_{j} T_{c}+b_{k} T_{\delta}$. Pulse Position Modulation (PPM) is assumed with $\left\{b_{k}\right\}$ being the information symbols taking values $\{0,1\}$ with equal probability. $p(t)$ refers to the single pulse waveform, being typically a Gaussian monocycle or one of its derivatives of duration $T_{p}$. $T_{\text {sym }}=N_{f} T_{f}$ is the symbol duration, where $T_{f}>T_{p}$ is the repetition pulse period also referred to as frame period, and $N_{f}$ is the number of frames per symbol, $T_{c}$ is the chip period, $T_{\delta}$ is the PPM modulation interval, $N_{c}$ is the number of chips per frame and $\left\{c_{j}\right\}$ is the time hopping sequence which takes integer values in $\left\{0,1, \ldots, N_{c}-1\right\}$.

The channel model considered is given by the general expression for the multipath fading propagation channel as follows,

$$
h(t)=\sum_{m=0}^{M-1} h_{m} \delta\left(t-\tau_{m}\right)
$$

where $h_{m}$ denotes the fading coefficient for the $\mathrm{m}$-th path while $\tau_{m}$ represents the delay experienced by the m-th path.

With no loss of generality we assume $\tau_{0}<\tau_{1}<\ldots<$ $\tau_{M-1}$, being $\tau_{0}$ the TOA that is to be estimated.

The received signal at the $\mathrm{q}$-th antenna element for a node equipped with an array of $\mathrm{Q}$ antenna elements is the summation of multiple delayed and attenuated replicas of the received pulse waveform $\widetilde{p}(t)$ which includes the antenna and filters distorsion,

$$
y_{q}(t)=\sum_{k=-\infty}^{\infty} \sum_{j=0}^{N_{f}-1} \sum_{m=0}^{M-1} h_{m} \tilde{p}\left(t-T_{j}^{k}-\tau_{m, q}\right)+v_{q}(t)
$$

where the temporal delay at each antenna element depends not only on the propagation delay but on the direction of arrival. In particular, for an uniform linear array (ULA), the propagation delay associated with the m-th arriving path in antenna $q$ is given by,

$$
\tau_{m, q}=\tau_{m}+q \frac{d}{c} \sin \theta_{m} \quad 0 \leq q \leq Q-1
$$

with $d$ being the distance between antenna elements in the array, $c$ the speed of light and $\theta_{m}$ the direction of arrival of the $m$-th path. Although the scheme is presented assuming an uniform linear array (ULA), it can be directly extended to other array configurations.

The additive noise $v_{q}(t) \sim N\left(0, N_{0}\right)$ is modeled as Gaussian circularly symmetric. Given the low duty cycle of UWB signals we assume the received signal is free of intersymbolic interference (ISI).

The signal associated to the $\mathrm{j}$-th transmitted pulse corresponding to the $\mathrm{k}$-th symbol at each antenna element, in the frequency domain yields:

$$
Y_{j q}^{k}(w)=\sum_{m=0}^{M-1} h_{m} S_{j}^{k}(w) e^{-j w \tau_{m, q}}+V_{j}^{k}(w)
$$

where $Y_{j q}^{k}(w), S_{j}^{k}(w)$ and $V_{j}^{k}(w)$ denote the Fourier transform of the received signal, transmitted signal and noise, respectively. Sampling and rearranging (5) into a matrix notation the received signal vector at the $q$ antenna element is given by,

$$
\mathbf{Y}_{j q}^{k}=\sum_{m=0}^{M-1} h_{m} \mathbf{S}_{j}^{k} \mathbf{e}_{\tau_{m, q}}+\mathbf{V}_{j}^{k}
$$

where the elements of vector $\mathbf{Y}_{j q}^{k} \in \mathbb{C}^{N \times 1}, Y_{j q}^{k}\left(w_{n}\right)$, are the DFT components of $y_{j q}^{k}(t)$ with $w_{n}=w_{0} n$ for $n=$ $0,1, \ldots, N-1$ and $w_{0}=2 \pi / N, \mathbf{S}_{j}^{k} \in \mathbb{C}^{N \times N}$ is a diagonal matrix whose elements are the DFT components of the transmitted signal and the noise samples are arranged in vector $\mathbf{V}_{j}^{k} \in \mathbb{C}^{N \times 1}$. The elements of the delay-signature vector include the dependency with the angle of arrival in $\tau_{m, q}$,

$$
\mathbf{e}_{\tau_{m, q}}=\left[\begin{array}{llll}
1 & e^{-j w_{0} \tau_{m, q}} & \ldots & e^{-j w_{0}(N-1) \tau_{m, q}}
\end{array}\right]^{T}
$$

With the aim of compact notation we consider a vector formed by the concatenation of the signals received at each antenna,

$$
\mathbf{Y}_{j}^{k}=\left[\begin{array}{llll}
\mathbf{Y}_{j, 0}^{k}{ }^{T} & \mathbf{Y}_{j, 1}^{k} & \ldots & \mathbf{Y}_{j, Q-1}^{k}
\end{array}\right]^{T}
$$

Consequently, vector $\mathbf{Y}_{j}^{k} \in \mathbb{C}^{Q N \times 1}$.

\section{JOINT TOA AND DOA ESTIMATION}

Estimation of the TOA from the noisy observation $y(t)$ without knowledge of the specific pulse shape $\tilde{p}(t)$ is the goal of this section. First, a simple coarse estimation stage that provides the time reference for symbol synchronization and estimates the threshold used in the TOA algorithm is needed and was presented in [9]. Then, a new joint fine TOA and DOA estimation algorithm is proposed. This two-stages estimation process is performed in the frequency domain. It is assumed that an all zero training sequence is used for the estimation. An acquisition time of length equal to the duration of $K_{s}+1$ symbols is considered. The TOA defined as $\tau_{0}$ indicates the beginning of the first complete symbol in the observation interval, being $0 \leq \tau_{0} \leq T_{\text {sym }}$. Note that the acquisition window duration is defined one symbol longer than the number of symbols considered for the fine timing estimation. Hence the minimum acquisition window shall be equivalent to two symbols duration in order to perform the fine estimation over a single symbol $K_{s}=1$.

Once the beginning of the symbol is coarsely estimated, working in the frequency domain any type of spectral estimation can be applied to obtain a two dimension power profile defined as, on the one hand, the signal energy distribution with respect to the propagation delays and, on the other hand, the signal energy distribution with respect to the angles of arrival.

The fine TOA estimation $\hat{\tau}_{0}$ is obtained from the TOA coarse estimation $\hat{\tau}_{0}^{c}$ and a high resolution time delay $\tilde{\tau}$ estimate of the first arriving path with respect to the time reference obtained in the coarse estimation stage. The TOA estimation resulting from the fine estimation stage is given by,

$$
\hat{\tau}_{0}=\hat{\tau}_{0}^{c}+\tilde{\tau}
$$


The fine TOA estimator consist of finding the first delay, $\tilde{\tau}$, that exceeds a given threshold, $P_{t h}$, in the power profile summed over all directions,

$$
\tilde{\tau}=\min \arg _{\tau}\left\{\sum_{\theta} P(\tau, \theta)>P_{t h}\right\}
$$

Once the first delay $\tilde{\tau}$ is obtained, the direction of arrival is estimated from the maximum of the power direction profile at $\tilde{\tau}$

$$
\tilde{\theta}=\arg \max _{\theta}\{P(\tilde{\tau}, \theta)\}
$$

The power profile can be obtained by estimating the energy of the signal filtered by the concatenation of the delaydirection signature vector, $\mathbf{E}_{\tau, \theta}$, at each time delay and each direction resulting in the quadratic form,

$$
P(\tau, \theta)=\mathbf{E}_{\tau, \theta}^{H} \mathbf{R E}_{\tau, \theta}
$$

where $\mathbf{R}$ is the signal correlation matrix. The signature vector $\mathbf{E}_{\tau, \theta}$ is formed by the signature vectors associated to each array element as,

$$
\mathbf{E}_{\tau, \theta}=\left[\begin{array}{llll}
\mathbf{e}_{0}^{T} & \mathbf{e}_{1}^{T} & \ldots & \mathbf{e}_{Q-1}^{T}
\end{array}\right]^{T}
$$

where,

$$
\mathbf{e}_{q}=\left(\begin{array}{llll}
1 & e^{-j w_{0}\left(\tau+q d \frac{\sin \theta}{c}\right)} & \ldots & e^{-j w_{0}(N-1)\left(\tau+q d \frac{\sin \theta}{c}\right)}
\end{array}\right)^{T}
$$

The correlation matrix $\mathbf{R}$ can be obtained averaging over $K_{s}$ symbols to obtain a more robust estimate and is defined as,

$$
\mathbf{R}=\frac{1}{N_{f} K_{s}} \sum_{k=1}^{K_{s}} \sum_{j=1}^{N_{f}} \mathbf{Y}_{j}^{k} \mathbf{Y}_{j}^{k^{H}}
$$

where $\mathbf{Y}_{j}^{k}$ is the frequency vector defined in (8).

The frequency domain samples $Y_{j q}^{k}[n]$ associated to the k-th symbol, $\mathbf{j}$-th frame and q-th antenna that form each vector $\mathbf{Y}_{j, q}^{k}$ are obtained from the time samples such that the monocycle positions are aligned,

$$
Y_{j q}^{k}[n]=\sum_{m=1}^{K_{f}} y\left[\hat{m}_{j}^{k}+m\right] e^{-j \frac{2 \pi}{K_{f}} n m} \text { for } n=1, \ldots, K_{f}
$$

being $\hat{m}_{j}^{k}$ the first sample associated to the $\mathrm{j}$-th transmitted pulse corresponding to the k-th symbol,

$$
\hat{m}_{j}^{k}=\left\lfloor\frac{\hat{\tau}_{0}^{c}+T_{j}^{k}}{T_{s}}\right\rfloor
$$

where $\hat{\tau}_{0}^{c}$ is the TOA coarse estimation, $T_{s}$ is the sampling period and $K_{f}=\left\lfloor T_{f} / T_{s}\right\rfloor$ is the number of samples in the frame period.

Note that the computation of the correlation matrix takes advantage of the inherent temporal diversity of the IR-UWB signal, with $N_{f}$ repeated transmitted pulses for each information symbol, by computing the correlation matrix over the $N_{f}$ received frames. This approach provides a better estimate of the correlation matrix and results in a more robust estimation algorithm, as compared to applying the estimator individually to each arriving frame.
TABLE I

SIMULATION PARAMETERS

\begin{tabular}{|c|c|}
\hline PARAMETER & VALUE \\
\hline \hline Pulse duration, $T_{p}$ & $0.4 \mathrm{~ns}$ \\
\hline Frame period, $T_{f}$ & $560 \mathrm{~ns}$ \\
\hline Pulse repetition Frequency, PRF & 20 \\
\hline Symbol period & $11.2 \mu \mathrm{s}$ \\
\hline Bandwidth & $2.5 \mathrm{GHz}$ \\
\hline
\end{tabular}

\section{NUMERICAL RESULTS}

For numerical evaluation of the algorithms we consider the channel models developed within the framework of the IEEE 802.15.4a. These models however do not explicitly include the spatial dependency when considering antenna array. Recent results [11] show that spectral anisotropy of UWB antennas may lead to localization ambiguities in systems that uses DOA measurements for positioning estimation. The paper also shows that using compensation techniques, DOA localization ambiguities can be removed. Given the lack of an standardized channel model that includes the spatial dimension, we follow the approach in [12] considering that the channel impulse response is a separable function in terms of time and azimuth angle dependency,

$$
h(t, \theta)=h_{a}(t) h_{b}(\theta)
$$

The dependency in time featured by $h_{a}(t)$ is generated according to [13] and the angular impulse response $h_{b}(\theta)$ is generated assuming a cluster model,

$$
h_{b}(\theta)=\sum_{l=0}^{\infty} \sum_{k=0}^{\infty} \beta_{k l} \delta\left(\theta-\Theta_{l}-\psi_{k l}\right)
$$

where $\beta_{k l}$ is the amplitude of $\mathrm{k}$-th arriving path in the 1th cluster, $\Theta_{l}$ is the mean azimuth angle-of-arrival of the 1th cluster and follows a uniform distribution and $\psi_{k l}$ is the azimuth angle-of-arrival relative to $\Theta_{l}$ and follows a zero mean Laplacian distribution with standard deviation, $\sigma$, of 38 degrees [12],

$$
p(\psi)=\frac{1}{\sqrt{2} \sigma} e^{-|\sqrt{2} \psi / \sigma|}
$$

The main simulation parameters are shown in Table I where the pulse waveform $p(t)$ is the Gaussian pulse.

All simulation results are given for 50 independent channel realizations. For each channel realization each antenna element sees a different channel according to the dependency on the direction of arrival. The signal bandwidth is of $2.5 \mathrm{GHz}$, centered at $f_{c}=7.25 \mathrm{GHz}$ according to the European ECC. The estimation is performed considering a single symbol $K_{s}=1$. The threshold level $P_{t h}$ used in the simulation is defined as the noise power level, which is estimated during the coarse estimation process.

Fig. 1 depicts the contour lines of a resulting periodogram using an array equipped with only two antennas in a LOS Residential scenario and with a single source located at 13 meters and 81.5 degrees. The actual periodogram is shown in 
Fig. 2 where a zoom into the area of interest has been made. For the scenario under evaluation, the actual time of arrival is $\tau_{0}=43.3 \mathrm{~ns}$ and $\sin (\theta)=0.989$. The estimated TOA and DOA are given by the ordinate and abscise values corresponding to the first maximum value of the periodogram, illustrated with a red dot in Fig. 2. From Fig. 1 it can be observed that for each multipath component there result associated contour lines with flat slope and contour lines that follow the slope subject to the antenna separation,

$$
\tau=\tau_{m}+q \frac{d}{c} \sin \left(\theta_{m}\right)
$$

The intersection of both slope-lines would determine the output estimate. The plot illustrates that the contribution to the periodogram for $\tau_{0}$ is maximum only in the right direction.

One may observe that the estimated TOA corresponds to the lowest value in the ordinates axis of Fig. 1, denoted as $\tau_{0}$, which represents the first arriving path.

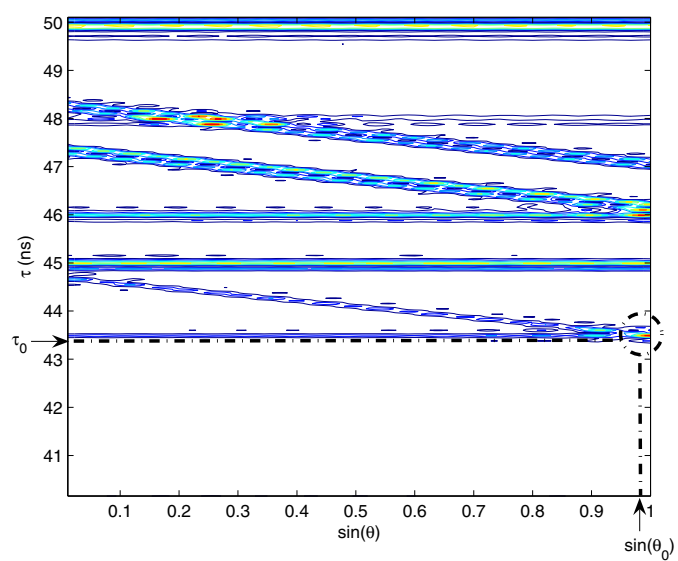

Fig. 1. Contour lines of the resulting periodogram for 2 element array with antenna elements separation of $36 \mathrm{~cm}$

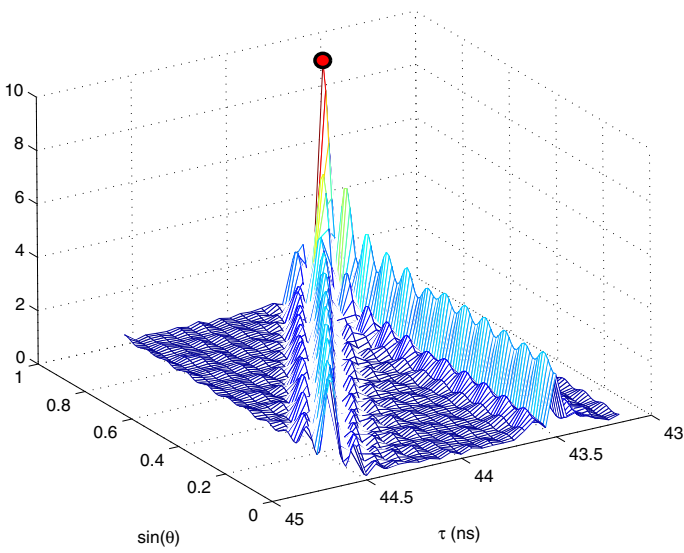

Fig. 2. Periodogram for 2 element array with antenna elements separation of $36 \mathrm{~cm}$

Fig. 3 depicts the root mean squared error (RMSE) of the estimated TOA for arrays equipped with two antennas, and an antenna spacing of $36 \mathrm{~cm}$, in a LOS Residential scenario (CM1 IEEE 802.15.4a channel). The results are compared to the Cramer-Rao bound derived for the joint TOA-DOA estimate [10]. Results show that the estimation accuracy for ranging applications is of the order of 2 centimeters.

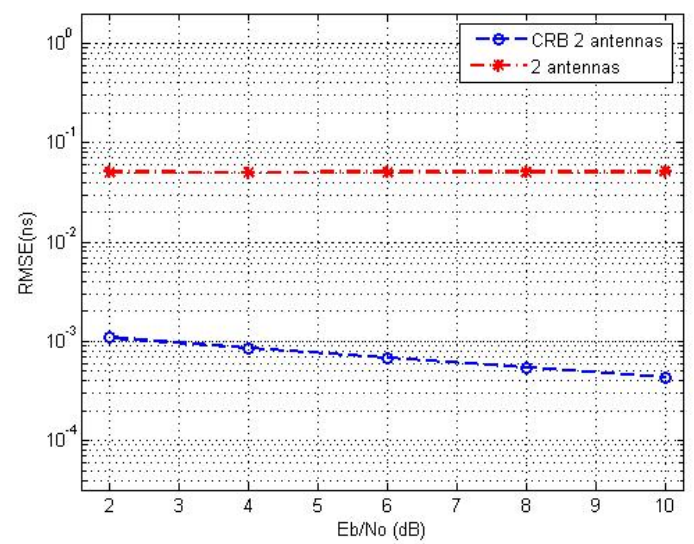

Fig. 3. Time of arrival $\tau_{0}$ estimation performance for 2 element array with antenna elements separation of $36 \mathrm{~cm}$

Likewise, Fig. 4 shows the estimated sine of the DOA for the same scenario. Shown for reference is the CRB computed as in [10].

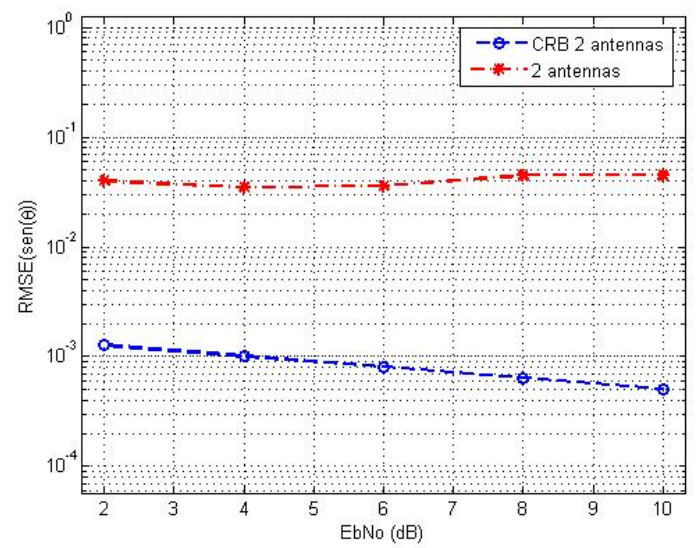

Fig. 4. Direction of arrival $\sin (\theta)$ estimation performance for 2 element array with antenna elements separation of $36 \mathrm{~cm}$

The algorithm has also been tested with 4 antenna elements maintaining the physical length of the array. Therefore, the spacing between antenna elements is reduced to $12 \mathrm{~cm}$. Results shown in Table II illustrate a slight improvement in the TOA estimation when the number of antennas increases (the estimated TOA is not sensitive to the separation between antenna elements). On the other hand, the estimation accuracy relative to the DOA, which from the CRB is expected to improve with the number of elements that form the array, in fact decreases due to the closer spacing between antennas. The antenna spacing will be subject to implementation constraints 
like the total array length and number of array elements. Nevertheless, the results have shown that good performance can be achieved with only two antennas.

TABLE II

EB/NO$=4 \mathrm{DB}$ ARRAYLENGTH $=36 \mathrm{CM}$

\begin{tabular}{|c|c|c|}
\hline ANTENNAS & RMSE(ns) & RMSE $(\sin (\theta))$ \\
\hline \hline 2 & $4.9696 \mathrm{e}-2$ & $3.4641 \mathrm{e}-2$ \\
\hline 4 & $4.7023 \mathrm{e}-2$ & $7.8740 \mathrm{e}-2$ \\
\hline
\end{tabular}

Finally, in Fig. 5 and 6 it is shown the RMSE of the estimated TOA and DOA respectively, for the case with two antennas and $\mathrm{Eb} / \mathrm{No}=4 \mathrm{~dB}$ as a function of antenna element separation. It can be seen that when the spacing between antennas increases the estimated DOA improves while the TOA estimation accuracy remains constant.

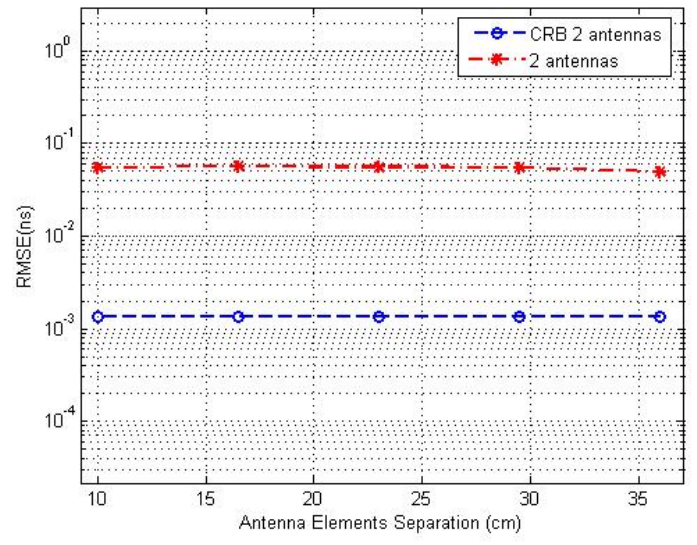

Fig. 5. Time of arrival $\tau_{0}$ estimation performance for 2 element array with $E b / N o=4 d B$

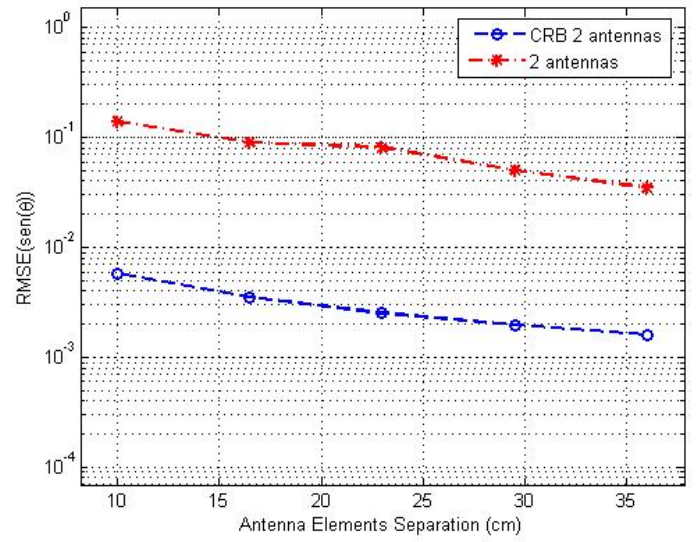

Fig. 6. Direction of arrival $\sin (\theta)$ estimation performance for 2 element array with $E b / N_{o}=4 d B$

We shall remark that the results depict directly the output of the estimator, that is the value of the sine of the DOA, rather than the angle itself. There is no need to transform to the angle domain for later application of TOA and DOA measurements in a positioning algorithm.

\section{CONCLUSION}

A joint TOA and DOA estimator for UWB systems has been presented. The estimation is performed in the frequency domain by computing the power delay-angle spectrum based on the periodogram quadratic form. The DOA estimation is able to exploit the high accuracy intrinsic to the large bandwidth of the UWB signal, since the angle estimation is performed from time delay measurements. Results have shown that only with two antenna element array accuracy of the order of $0.1 \mathrm{~ns}$ can be achieved for TOA estimation, while DOA root mean squared error remains below 10 degrees. Performance of the TOA estimator obtained by simulation agrees with the $\mathrm{CRB}$, showing no dependency of the RMSE with the antenna separation. Opposite to DOA estimation, where both numerical results and $\mathrm{CRB}$ show a decrease on the RMSE with the antenna separation.

\section{REFERENCES}

[1] J.J. Blanz, A. Papathanassiou, M. Haardt, I. Furio, and P.W. Baier, "Smart antennas for combined DOA and joint channel estimation in time-Slotted CDMA mobile radio system with joint-detection", in IEEE Trans. Veh. Technol., vol.49, pp. 293-306, Mar. 2000.

[2] Y.Y. Wang, J.T. Chen and W.H. Fang, "TST-MUSIC for joint DOA delay estimation", in IEEE Trans. Signal Processing, vol.49, pp. 721-729, Apr 2001.

[3] M. Cedervall and A. Paulraj, "Joint channel ans space-time parameter estimation", in 30th Asilomar Conf. Signals, Systems, Comput., vol.1, Nov. 1996, pp. 375-379.

[4] M.C. Vanderveen, A. Van der Veen, and A. Paulraj, "Estimation of multipath parameters in wireless communications", in IEEE Trans. Signal Processing, vol.46, pp. 682-690, Mar. 1998.

[5] M. Wax and A. Leshem, "Joint estimation of time delays and directions of arrival of multiple reflections of a known signal", in IEEE Trans. Signal Processing, vol.45, pp. 2477-2428, Oct. 1997.

[6] N. Hew and N. Zein "Space-time Estimation Techniques for UTRA System", in IEE Colloquium on Capacity and Range Enhancement Techniques for the Third Generation Mobile Communications and Beyond, Ref. No. 2000/003, pp. 6/1-6/7, Feb. 2000.

[7] J.P. Lie, B.P. Ng, C.M. See and MA. Changzheng, "Multiple UWB Emitters DoA Estimation Employing TH-SS",in IEEE Inter. Conf. on Wireless Communications, Networking and Mobile Computing, Vol.1, 2326 Sept. 2005 pp. 336 - 339.

[8] J.P. Lie, C.M. See and B.P. Ng, "Ultra WideBand Direction Finding using Digital Channelization Receiver Architecture", in IEEE Communication Letters, Volume 10, pp. 85-87, Feb 2006.

[9] M. Najar and M. Navarro, "Joint synchronization and demodulation for IR-UWB", in IEEE International Conference on Ultra-Wideband, Hannover, Germany, Sept. 10-12 2008. (ICUWB2008)

[10] M. Navarro and M. Najar, "Frequency Domain Joint TOA and DOA estimation in IR-UWB", submitted to the IEEE Transactions on Wireless Communications, Oct. 29, 2008.

[11] W.Q. Malik,C.J. Stevens and D.J. Edwards, "Ultrawideband Antenna Distortion Compensation", in IEEE Transactions on Antennas and Propagation, Vol. 56, No.7, pp. 1900-1097, July 2008.

[12] R.J.-M. Cramer,R. Scholtz and M. Win, "Evaluation of an ultra-wideband propagation channel", in IEEE Transactions on Antennas and Propagation, Vol. 50, No.5, pp. 561-570, 2002.

[13] A.F. Molisch,K. Balakrishnan, C. Chong, S. Emami, A. Fort, J. Karedal, J. Kunisch, H. Schantz, U. Schuster and K. Siwiak, "P802.15-04-066200-004a, ieee 802.15.4a channel model - final report”, 2004. 